\title{
Besenheide (Calluna vulgaris), Blume des Jahres 2019
}

\author{
Veit Martin Dörken \& Armin Jagel
}

\begin{abstract}
The common heather (Calluna vulgaris) is flower of the year 2019. Different species of the genera Calluna and Erica are described as heather. Especially Calluna vulgaris is an important ornamental plant for the garden. Of horticultural importance is budding heather. Their winter-appearing buds are of great ornamental value. Nonetheless they do not offer food to insects.
\end{abstract}

\section{Zusammenfassung}

Die Besenheide (Calluna vulgaris) ist Blume des Jahres 2019. Unter Heide versteht man verschiedene Arten vor allem der Gattungen Calluna und Erica. Calluna vulgaris ist eine wichtige Zierpflanze für den Garten. Von gärtnerischer Bedeutung sind Knospenheiden. Ihre im Winter erscheinenden Knospen haben einen großen Zierwert. Sie bieten aber Insekten keine Nahrung

\section{Einleitung}

Für das Jahr 2019 hat die Loki-Schmidt-Stiftung die Besenheide zur Blume des Jahres ausgerufen, um auf den Lebensraum Heide und seine Gefährdung hinzuweisen. Die Wahl irritiert zunächst ein wenig, da man sich unter einer Blume normalerweise eine rein krautige Pflanze vorstellt und keinen verholzten Zwergstrauch, wie es die Besenheide ist. Aber vielleicht sind diese Vorbe- halte auch zu „akademisch“ und viele Menschen verstehen unter einer Blume einfach nur eine blühende Pflanze. Unter den seit 1980 ausgerufenen „Blumen des Jahres“ war auch schon im Jahr 1991 ein Zwergstrauch dabei, nämlich die Rosmarinheide (Andromeda polifolia). Unter den Rubriken fehlt der "Strauch des Jahres“, so dass die Besenheide als Zwergstrauch am besten in die Rubrik „Blume“ passt.

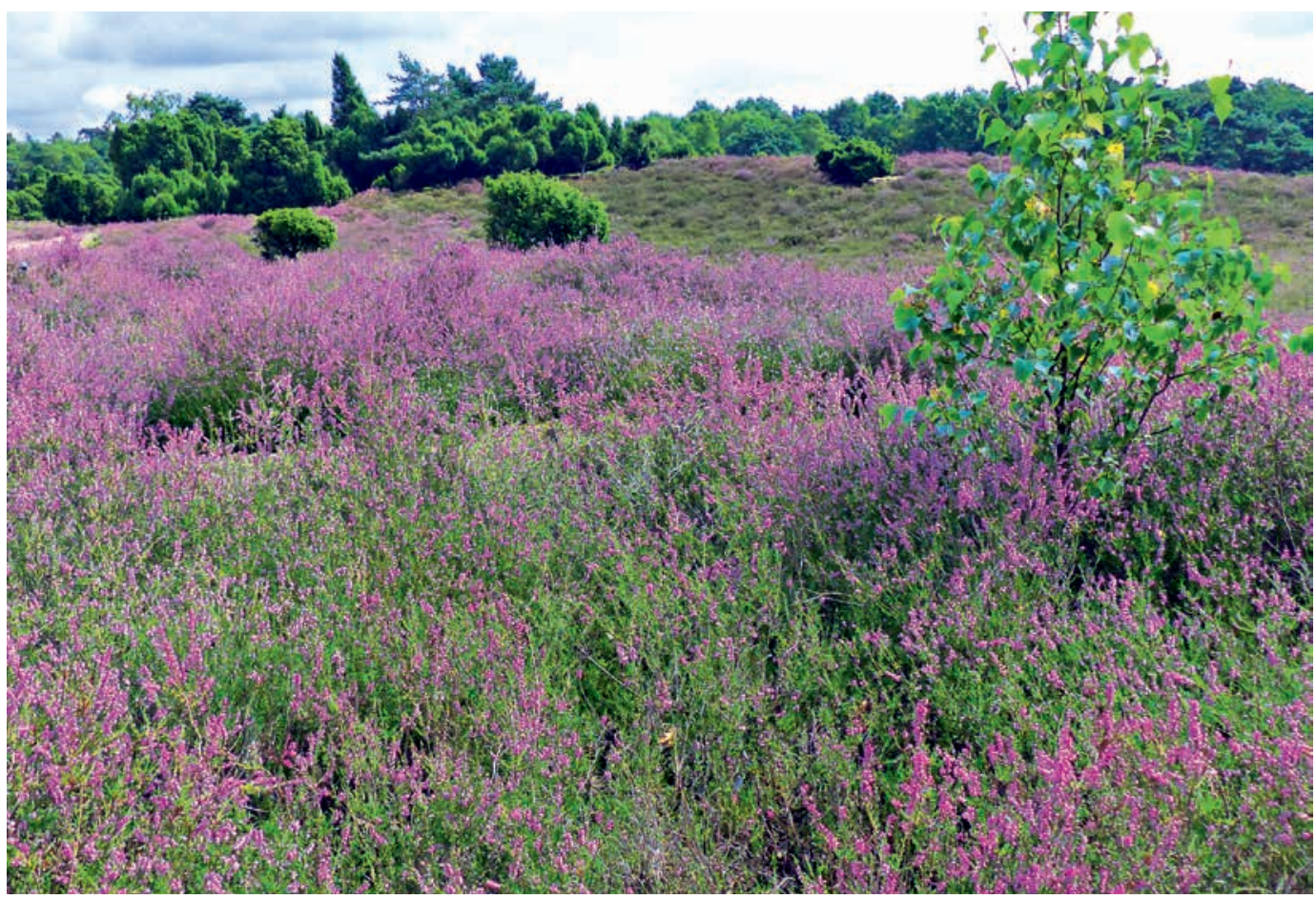

Abb. 1: Heidelandschaft mit blühender Calluna vulgaris in der Westruper Heide in der Nähe des Halterner Stausees.

(Foto: H. STEINecke) 


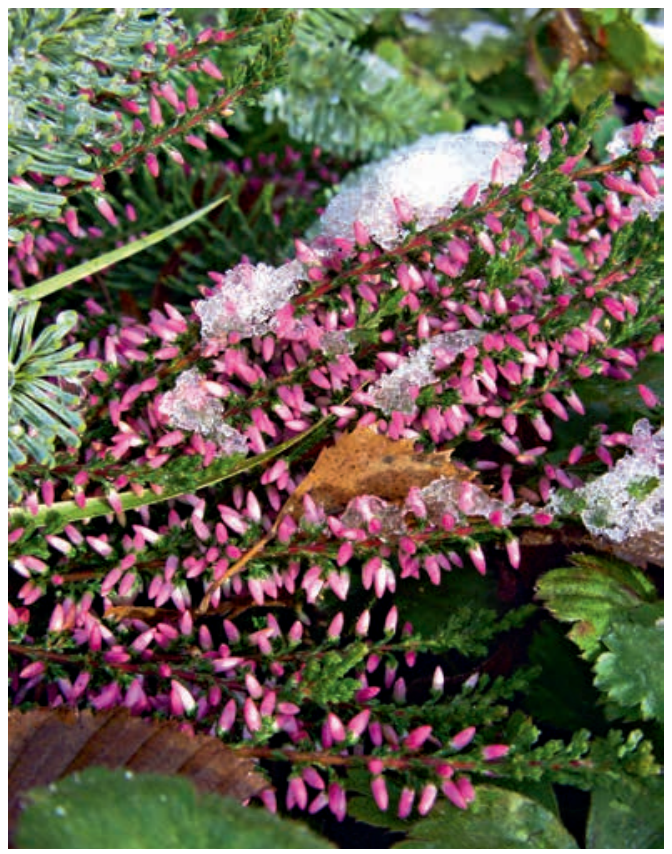

Abb. 2: Knospenblütige Besenheide (Calluna vulgaris). (Foto: A. JAGEL)

Im Herbst wird im Gartenhandel massenhaft „Heide“, „Erika“ oder auch „Heidekraut“ zur Bepflanzung von Gräbern, Kübeln oder Gärten angeboten. Die Handelsbezeichnungen nehmen in den meisten Fällen keinen Bezug auf die tatsächliche Art. Botanisch handelt es sich dabei um Arten aus zwei verschiedenen Gattungen aus der Familie der Heidekrautgewächse (Ericaceae): Calluna und Erica. Morphologisch lassen sie sich relativ leicht voneinander unterscheiden. Bei den für Herbst- und Winterbepflanzung verwendeten Arten handelt es sich im Wesentlichen um Sorten der Besenheide (Calluna vulgaris), der Schneeheide (= Winterheide, Erica carnea $=$ E. herbacea $)$ und der Englischen Heide (Erica $\times$ darleyensis), die aufgrund ihrer späten bzw. sehr frühen Blütezeit besonders gut für die Winterzeit geeignet sind. Die beiden erstgenannten Arten gehören zur Flora Deutschlands. Gepflanzt werden aber in der Regel nicht die Wildformen, sondern Zuchtformen, wobei man mit den sogenannten Knospenheiden beim Heidekraut Sorten selektiert hat, die von besonders hohem gärtnerischem Wert sind.

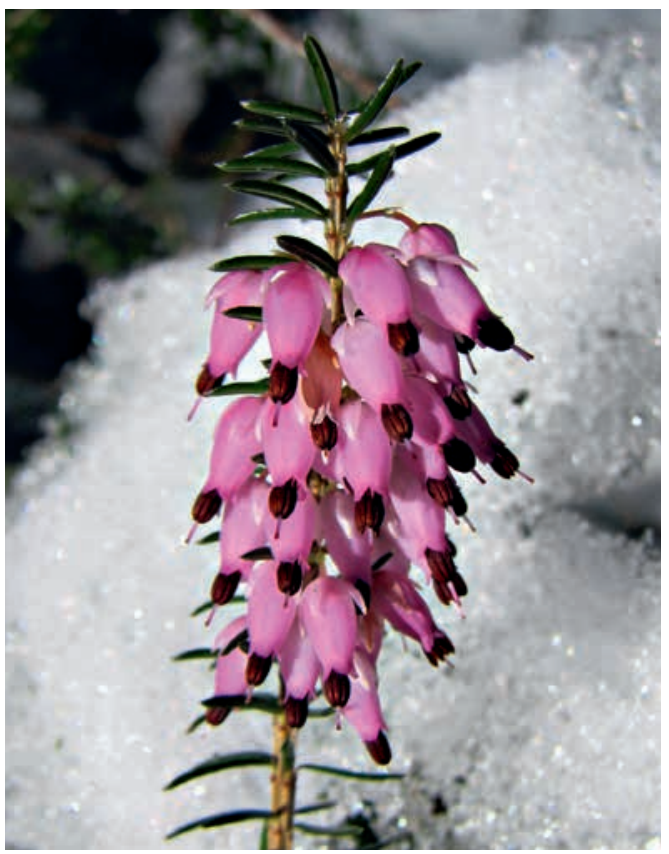

Abb. 3: Die Schneeheide (Erica carnea) blüht, wie der Name erahnen lässt, im Winter und auch bei Schnee. (Foto: A. JAGEL)

\section{Bedeutung des Namens}

Der deutsche Name „Besenheide“ für Calluna vulgaris leitet sich von der früheren Verwendung der Zweige für die Besenherstellung ab. Auch die botanische Bezeichnung Calluna bezieht sich darauf, denn das aus dem Griechischen abgeleitete kalynein bedeutet so viel wie schön machen bzw. reinigen. Die Vokabel Heide ist auf die germanische Sprache zurückzuführen. Man bezeichnete damit ein unbebautes Land (Kait). Dieses Wort entwickelte sich im Althochdeutschen dann zu Heida und schließlich zu Heide. Die Gattung Erica wird volkstümlich ebenfalls als Heide oder Heidekraut bezeichnet. Der Name entstammt dem Griechischen (Ereike) und bezeichnet die im Mittelmeergebiet heimisch Baum-Heide ( $E$. arborea, vgl. Genaust 2005).

\section{Systematik}

Calluna sowie Erica sind Heidekrautgewächse (Ericaceae). Diese große Pflanzenfamilie umfasst weltweit über 117 Gattungen mit knapp 4000 Arten. $\mathrm{Zu}$ den Ericaceae gehören auch andere bei uns beliebte Zierpflanzen aus den Gattungen Rhodo- 


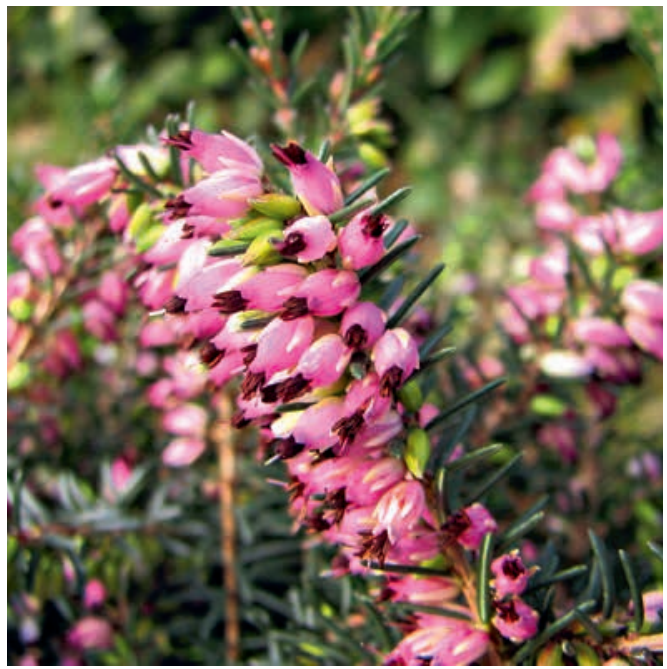

Abb. 4: Erica $\times$ darleyensis 'Kramers Rote'. (Foto: V. M. DÖRKEN)

dendron (Alpenrose), Vaccinium (Heidel-, Preiselund Moosbeere), Enkianthus (Prachtglocke) und Daboecia (Irische Heide, Glanz-Heide). Die Gattung Calluna umfasst nur eine einzige Art. Erica dagegen ist mit weltweit etwa 640 Arten eine große Gattung. In Deutschland sind nur drei Erica-Ar-

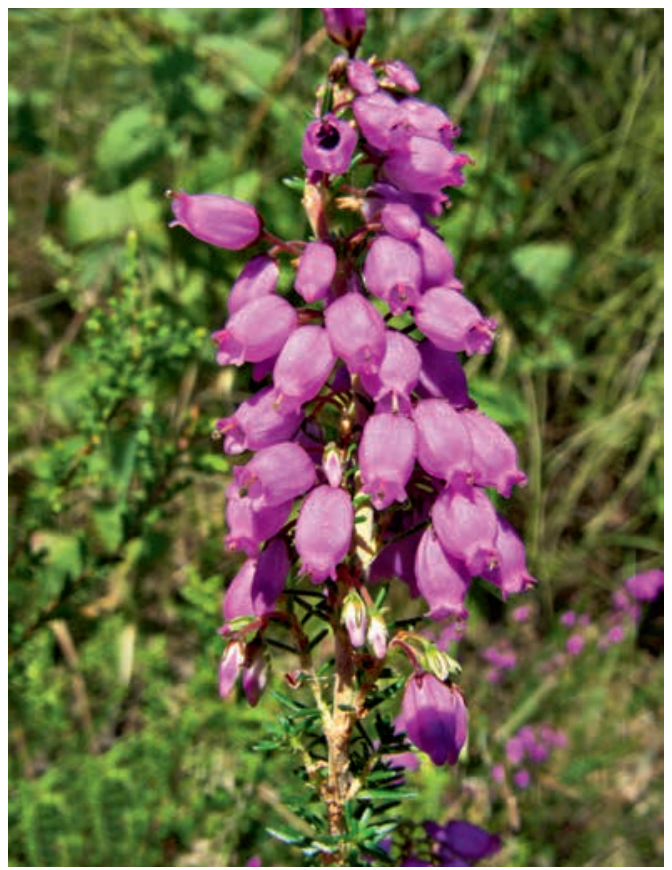

Abb. 5: Erica cinerea. (Foto: A. JAGEL) ten heimisch: die sehr seltene Grau-Heide (Erica cinerea), die weit verbreitete Glocken-Heide (Erica tetralix) und die Schnee-Heide (E. carnea), die überwiegend im Alpenraum, aber auch in manchen Mittelgebirgen wie dem Fichtelgebirge wild wächst. Wegen ihrer Blütezeit im Winter ist die Schnee-Heide als Gartenpflanze besonders beliebt.

\section{Verbreitung und Lebensraum}

Die Besenheide (Calluna vulgaris) hat ein großes Gesamtareal von den Azoren bis zum Ural und dringt vom nördlichen Kleinasien bis in den Norden Marokkos (Roloff \& Bärtels 1996). Auch in Deutschland ist sie weit verbreitet. Im Flachland kommt sie besonders in den Heidegebieten und auf offenen, bodensauren Standorten vor. Im Bergland trifft man auf sie vor allem an offenen, hellen Standorten in bodensauren Wäldern und in den noch verbliebenen Hochheiden.

Die Schneeheide ist in den Alpen ein häufiger, immergrüner Zwergstrauch, der mitunter ausgedehnte Bestände bildet. Sie ist ein typisches Element der alpinen Kiefernwälder und Latschengebüsche und eine Kennart des Schneeheide-Kiefernwaldes. Darüber hinaus kommt sie aber auch auf Schotterterrassen alpiner Flüsse vor (Düll \& Kutzenigg 2011). Im Gegensatz zu den meisten Erica-Arten ist Erica carnea auch auf kalkhaltigen Substraten wie Dolomit oder Kalkgestein anzutreffen.

\section{Morphologie}

\subsection{Habitus}

Besenheide sowie auch die beiden anderen EricaArten sind immergrüne Gehölze. Die in Mitteleuropa heimischen Arten sind Zwergsträucher bis 50 Zentimeter Höhe. Sie neigen im Alter zum Verkahlen. Im Gegensatz zu Calluna-Arten verkahlen Erica-Arten nicht so stark. Ältere Calluna-Pflanzen sind nur noch in ihrem oberen Bereich beblättert. Daher ist bei Gartenpflanzen ein jährlicher Rückschnitt notwendig, um dieses Verkahlen zu verhindern und damit einen üppigere Wuchsform zu erhalten. In den noch verbliebenen Heideflächen erfolgt die großflächige Verjüngung 


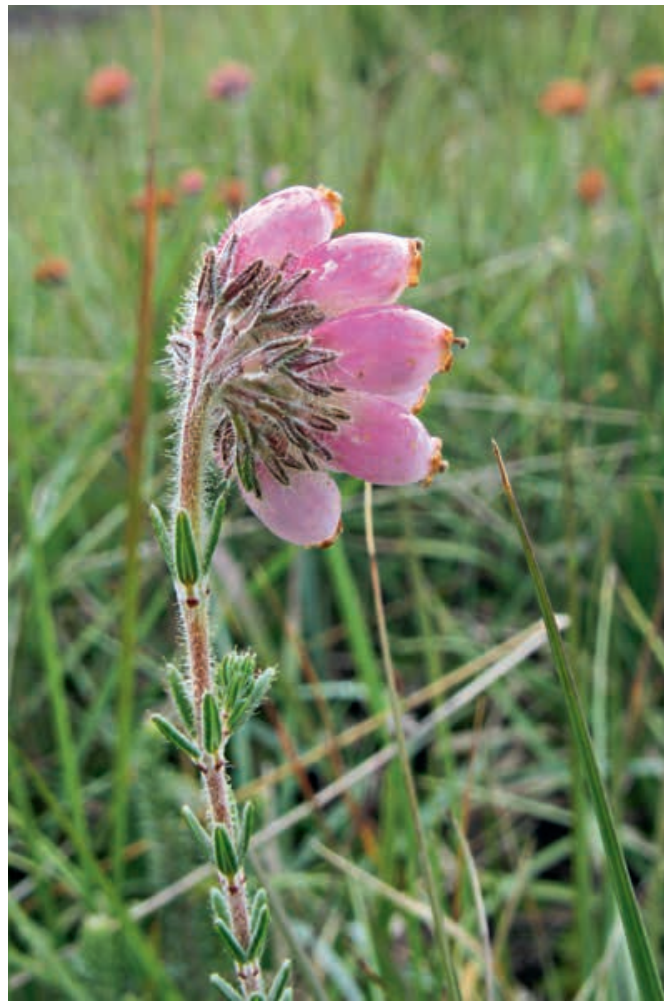

Abb. 6: Erica tetralix in der Wahner Heide bei Troisdorf. (Foto: A. JAGEL)

durch Beweidung mit Schafen wie den berühmten Heidschnucken der Lüneburger Heide. Verkahlte Pflanzen wurden früher geschnitten, das Reisig dann zur Herstellung von Besen verwendet.

\subsection{Blatt}

Die Blätter der Besenheide sind schuppenförmig. Sie stehen gegenständig und liegen meist dem Spross etwas an, sodass sie sich dachziegelartig überdecken. An der Basis haben sie einen zweizipfeligen Sporn. Die Blattränder sind mehr oder weniger stark eingerollt, weshalb sie als Rollblätter bezeichnet werden. Die Spaltöffnungen sind zwischen zahlreichen Papillen auf der Blattunterseite konzentriert.

Derartig kleine Blätter haben sich bei vielen Pflanzenarten als Anpassung an Trockenheit gebildet, um die wasserabgebende Oberfläche zu reduzieren. Bei Calluna ist die Reduktion der Blattfläche als Anpassung an den nährstoffarmen

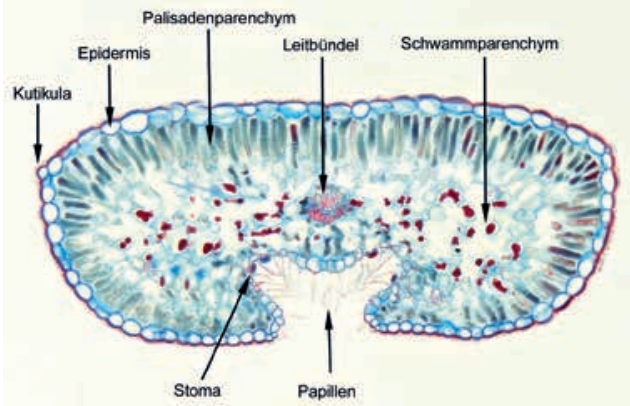

Abb. 7: Querschnitt durch das Blatt von Calluna vulgaris. (Foto: V. M. DörkeN)

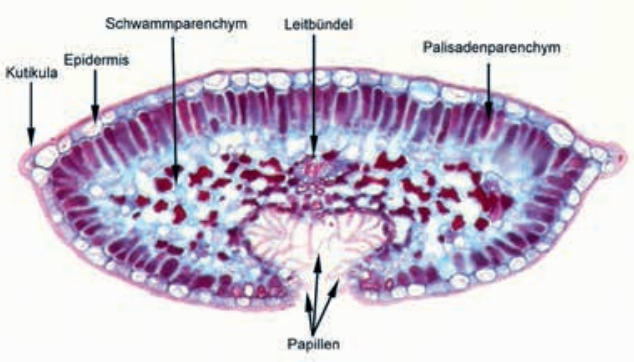

Abb. 8: Querschnitt durch das Blatt von Erica $\times$ darleyensis 'Kramers Rote'. (Foto: V. M. DöRKeN)

(Stickstoffmangel) Standort zu verstehen (Peinomorphose, vgl. Düll \& Kutzelnigg 2016). Abgefallene Blätter des Heidekrauts sind wie auch die übrigen Pflanzenteile schlecht abbaubar. So gilt Besenheide aufgrund der massenhaften Produktion von Rohhumus als Bodenverschlechterer.

Im Gegensatz zu Calluna sind die Blätter von Erica nadelartig und sitzen zu 3-4 (manchmal auch mehr) am Spross quirlständig. Sie stehen deutlich, oft sogar rechtwinklig, ab. Der Blattrand ist ebenfalls meist zur Blattunterseite hin eingerollt, auch wenn dies äußerlich kaum zu erkennen ist, sodass sie im Querschnitt denen von Calluna sehr ähnlich sind. Die Blattspitzen sind häufig spitz und stechend.

\subsection{Blüte}

Die Blütezeit der Besenheide liegt bei uns zwischen August und Oktober. Die Blüten befinden sich in langen Blütenständen am Zweigende. Die Ein- 


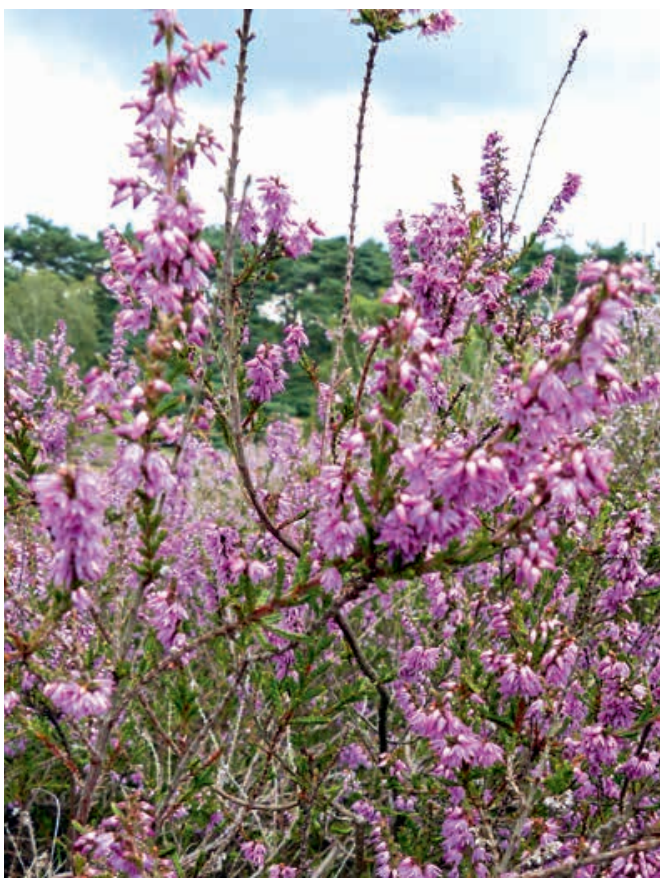

Abb. 9: Blühende Besenheide (Calluna vulgaris).

(Foto: H. Steinecke)

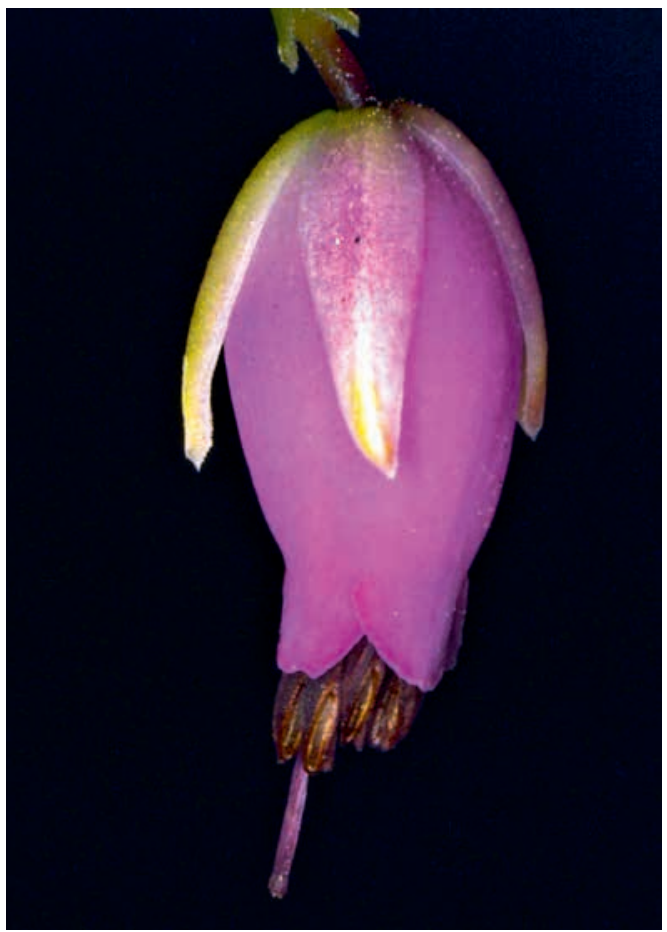

Abb. 10: Kegelförmige Erica $\times$ darleyensis-Blüte. (Foto: V. M. DöRKEN) zelblüte hat vier gleichfarbene Kelch- und Kronblätter. Die Schauwirkung der Blüte übernehmen die Kelchblätter, die wesentlich größer sind als die Kronblätter und diese deutlich überragen (Unterschied zu Erica). Die unscheinbare Blütenkrone der Besenheide ist trockenhäutig und bleibt nach der Blütezeit noch lange erhalten.

Anders als das Heidekraut ist die Schnee-Heide ein echter Winterblüher, dessen Blütezeitraum sich von Dezember bis April erstreckt. Die Krone der rosafarbenen Einzelblüten ist, wie für Erica typisch, glockenförmig ausgebildet, die Blüten nicken. Die Mündung der Krone ist dabei verengt. Zum Blütezeitpunkt ragen die Staubblätter wie auch der Griffel mit der Narbe weit aus der Blüte heraus.

Die Bestäubung erfolgt hauptsächlich durch Bienen (Produktion von „Heidehonig“) oder Falter. Die Staubbeutel bilden einen Streukegel aus und öffnen sich bereits in der Knospe. Dort geben sie den trockenen Pollen in den

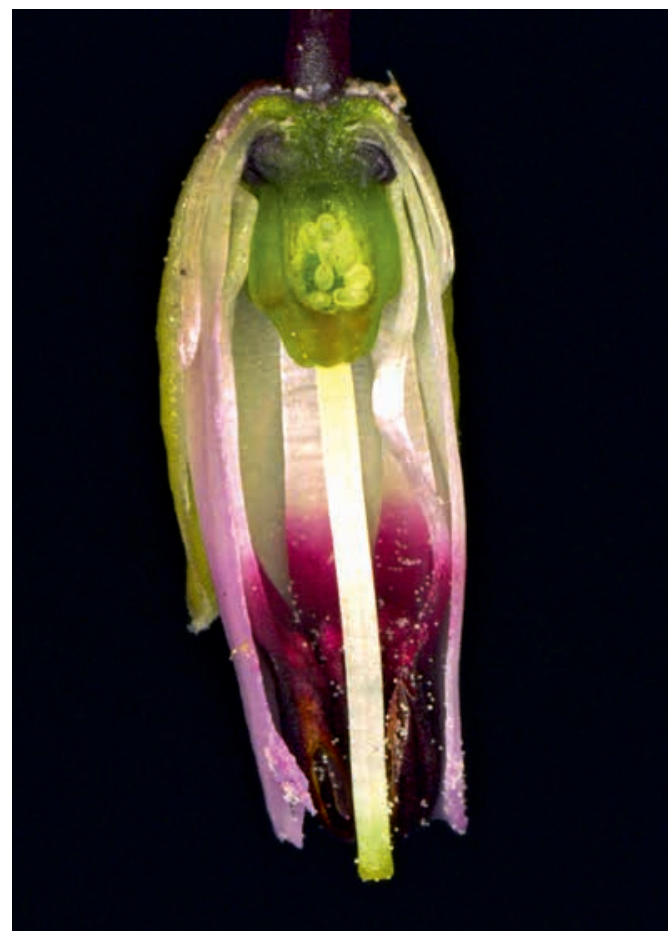

Abb. 11: Längsschnitt durch eine Erica $\times$ darleyensis-Blüte. (Foto: V. M. DörkeN) 


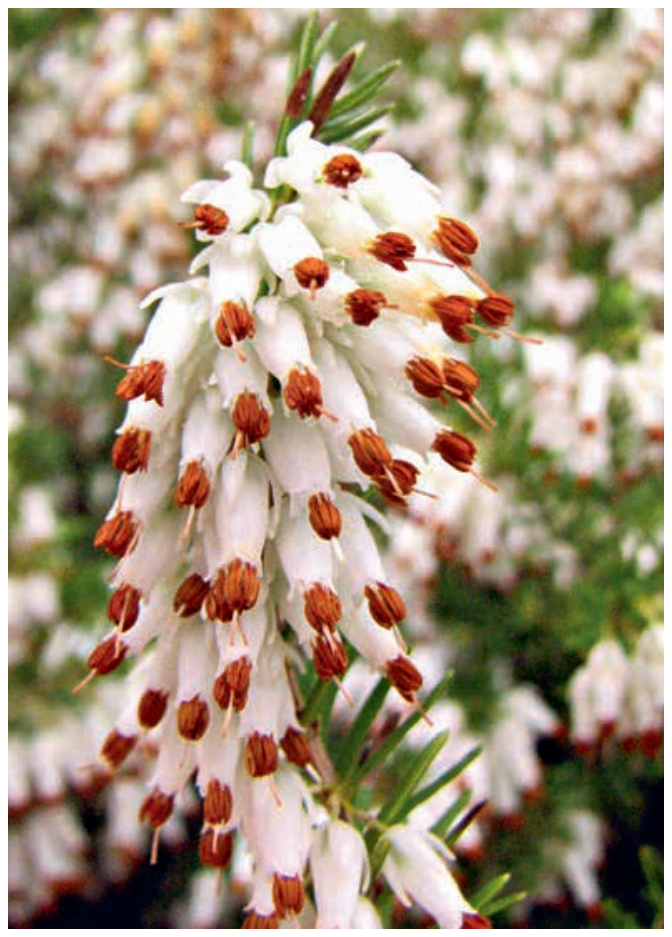

Abb. 12: Erica carnea 'Snowwhite'. (Foto. V. M. DöRkEN)

Kegel ab (sogenannter Streukegelmechnismus). Das Loch in der Spitze dieses Streukegels wird durch den Griffel verschlossen. Wenn ein Insekt beim Blütenbesuch an den Griffel stößt, fällt der Pollen aus dem Kegel wie aus einem Salzstreuer auf den Bestäuber herab. Sollte einmal der Insektenbesuch ausbleiben, kommt es zum verstärkten Wachstum der Staubfäden und der Pollen wird durch den Wind ausgebreitet (DüLL \& Kutzelnigg 2016).

\subsection{Frucht}

Bei beiden Arten sind die Früchte rundliche Kapseln, die sich mit Klappen öffnen. Sie enthalten zahlreiche kleine schwarze Samen. Bei Calluna bleibt die Frucht von den Kelchblättern größtenteils umhüllt. Die Fruchtreife erfolgt hier im Frühjahr des kommenden Jahres. Ähnlich wie beim Mohn (Papaver) werden die Samen aus der reifen Frucht durch den Wind ausgeschüttelt. Laut Düll \& Kutzelnigg (2005) fördern Brände bei Calluna die Samenkeimung deutlich. Als Pionierart brauchen die Samen Licht zum Keimen.

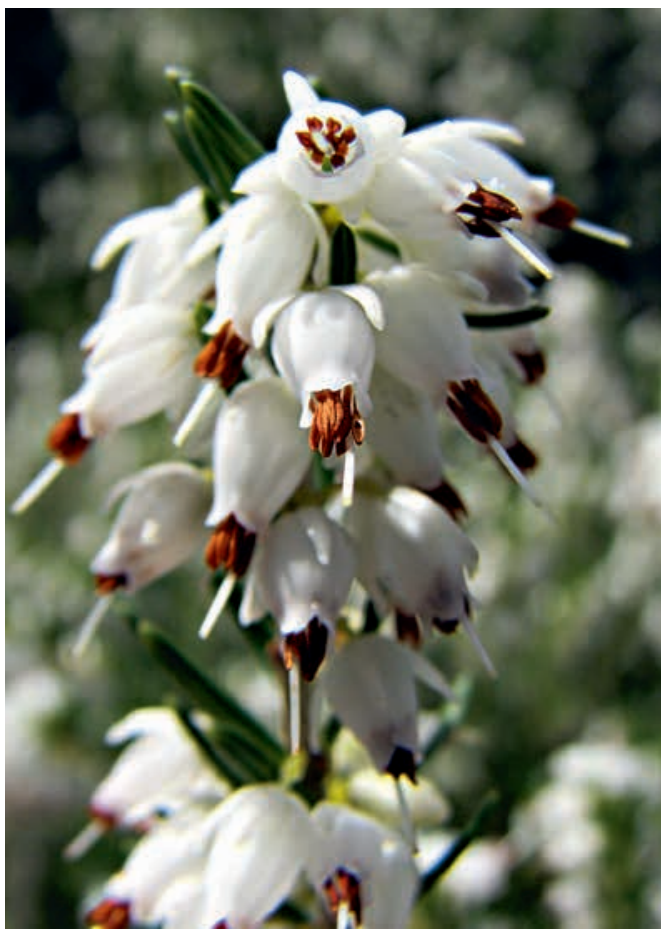

Abb. 13: Erica $\times$ darleyensis 'White Perfection'.

(Foto: V. M. Dörken)

\section{Sorten}

Bei der Besenheide sind durch gärtnerische Auslesen mittlerweile mehr als 1000 Sorten entstanden. Bei der Zucht kam es einerseits auf verschiedene Blütenfarben (weiß über rosa bis dunkelviolett) und die Farbe der Blätter an. Andererseits war ein wichtiges Ziel, den Blütezeitpunkt der Art nach hinten zu verlagern, denn der natürliche Blütezeitpunkt der Besenheide liegt natürlicherweise nicht im Winter (s. o.). So entstanden die sogenannten Knospenheiden (Knospenblüher), die heute von größter gärtnerischer Bedeutung sind. Solche knospenblütigen Pflanzen bringen eine große Fülle kleiner farbiger Knospen hervor, die eine hervorragende Schauwirkung haben, obwohl sich die Blüten gar nicht öffnen. Die farbigen Knospen selbst verharren praktisch den ganzen Winter in der Knospenruhe, weswegen sie nicht verwelken und ihr Zierwert bis in den März erhalten bleibt. So wurde das ab dem Herbst blühende Heidekraut zu einem unserer bekanntesten Winterblüher der Gärten und Friedhöfe, ohne dass sie aber einem Blütenbesucher etwas nützen. 


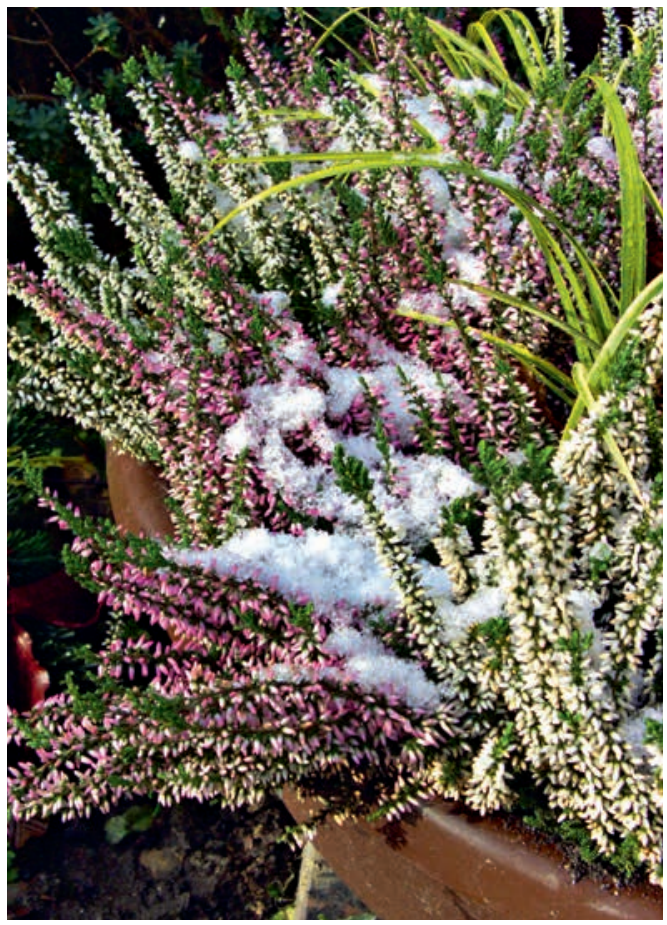

Abb. 14: Verschiedenfarbige Sorten einer knospenblütigen Besenheide (Calluna vulgaris). (Foto: V. M. DöRkeN)

Anders als das Heidekraut ist die Schnee-Heide ein echter Winterblüher. Aber auch hier sind die Blütenknospen bereits im Herbst vollständig ausgebildet, sodass sie einen gewissen Zierwert bereits vor der Blütezeit haben. Auch von dieser Art gibt es zahlreiche Sorten, deren Farbspektrum der Blüten von purpurfarben über rosa bis weiß reicht.

Neben den beiden genannten Arten und ihren Sorten spielt auch die sogenannte Englische Heide (Erica $\times$ darleyensis) im Gartenhandel eine große Rolle. Hierbei handelt es sich um eine Hybride zwischen der Schnee-Heide (Erica carnea) und der aus Westeuropa (Irland, Frankreich, Spanien) stammenden Purpur-Heide (E. erigenam, Roloff \& Bärtels 2008). Die Englische Heide ähnelt im Aussehen sehr stark der Schnee-Heide und wird häufiger auch als solche verkauft. Im Vergleich zur Schnee-Heide werden die Pflanzen höher (z. T. bis 90 Zentimeter), haben längere Blätter und, besonders wichtig für den Zierwert, blühen früher und länger. Die Blütezeit beginnt manchmal schon Ende Oktober/Anfang November und kann bis Mai anhalten. Allerdings ist die Englische Heide weniger winterhart als die Schnee-Heide (KöHlein et al. 2000).

Kurzum, Heidekräuter sind in verschiedensten Varianten über viele Monate hinweg attraktive Zierpflanzen. Heide als Sammelbegriff gehört zu den wenigen Pflanzen, die selbst von botanisch nicht versierten Menschen auf Anhieb erkannt werden. Schließlich sind Erika und Heide (davon abgeleitet auch Heidrun, Heidemarie, Adelheid, Heiderose) auch althergebrachte deutsche weibliche Vornamen.

\section{Literatur}

Düll, R. \& Kutzelnigg, H. 2011: Taschenlexikon der Pflanzen Deutschlands. Ein botanisch-ökologischer Exkursionsbegleiter, 7. Aufl. - Wiebelsheim.

Genaust, H. 1996: Etymologisches Wörterbuch der botanischen Pflanzennamen, 3. Aufl. - Hamburg.

Haeupler, H., Jagel, A. \& Schumacher, W. 2003: Verbreitungsatlas der Farn- und Blütenpflanzen in Nordrhein-Westfalen. LÖBF NRW (Hrsg.). - Recklinghausen.

Köhlein, F., Menzel, P. \& Bärtels, A. 2000: Das große Ulmer-Buch der Gartenpflanzen. - Stuttgart.

Mabberley, D. J. 2008: Mabberley's plant book, 3. Aufl. Cambridge.

Roloff, A. \& Bärtels A. 2008: Flora der Gehölze. - Stuttgart.

\section{Anschriften der Autoren:}

Dr. Veit Martin Dörken, Universität Konstanz, FB Biologie, Universitätsstraße 10, 78457 Konstanz, E-Mail: veit.doerken@uni-konstanz.de

Dr. Armin Jagel, Danziger Str. 2, 44789 Bochum, E-Mail: armin@jagel.nrw 